\title{
Dickkopf-1 ou un candidat têtu à l'induction de la tête
}

Spemann et Mangold avaient montré dans les années 1920 que le centre organisateur (dit de Spemann) chez l'amphibien peut être subdivisé en tissus capables d'induire la formation du tronc ou de la tête. Cette dichotomie physique des potentialités d'induction de l'organisateur indiquait que les mécanismes moléculaires de l'induction de la tête et du tronc pourraient être découplés chez les vertébrés. Dans l'analyse de la spécification des axes du plan de l'organisme, les mécanismes organisateurs du tronc ont été largement étudiés. En particulier, plusieurs facteurs sécrétés par le centre de Spemann ont été caractérisés et leur identification a permis de confirmer la proposition originale de Spemann, selon laquelle l'organisateur des vertébrés devait être subdivisé en domaines séparés induisant la tête et le tronc [1]. Les signaux organisateurs de la tête sont moins bien connus que ceux $\mathrm{du}$ tronc, bien que plusieurs gènes candidats à l'induction de structures antérieures aient été décrits ces dernières années. Il semble qu'on s'avance résolument vers l'élucidation de cette question fondamentale du développement, à l'aide du modèle incontournable du xénope mais aussi de la souris après les récentes caractérisations des gènes Dickkopf, ou $d k k-1$, et $m$-Cerberus-like [2, 3].

\section{$d k k-1$, inducteur de la tête chez le xénope}

L'induction des structures antérieures chez le xénope, telles que la tête et les glandes adhésives, réclame un agent spécifique, actif même en l'absence de formation de mésoderme.

C'est par criblage génique différentiel que l'équipe de Niehrs (Heidel-
Dickkopf (dkk-1) [2]. Exprimé dans le centre organisateur de Spemann, il code pour une nouvelle protéine sécrétée, à deux domaines riches en cystéines, capable d'induire la formation de la tête et d'axes secondaires complets. Ces propriétés antériorisantes sont observées lorsque l'ARN messager de $d k k-1$ est injecté dans l'embryon avec celui de la forme dominante négative du récepteur des BMP2/4 (tBR) qui inhibe la voie de transmission du signal des BMP [2]; à l'inverse, l'injection d'anticorps anti-dkk dans l'embryon provoque des «troncations» de la tête: la présence de dkk-1 serait donc nécessaire à sa formation. Rappelons que la protéine BMP4, de la famille du TGF $\beta$, inhibe la spécification neurale et que son inhibition est nécessaire à l'induction du cerveau ( «le cerveau par défaut») [4]. Les gènes $t B R$ et $d k k 1$ agissent en synergie pour induire l'expression de gènes inhibiteurs de la voie Wnt: goosecoid, chor$\operatorname{din}$ et $\operatorname{otx} 2 *$. Ces facteurs interagiraient directement avec les protéines Wnt ou leurs récepteurs mais avec des affinités différentes. De ces facteurs inducteurs de tête, dkk-1 est le plus puissant, le plus antériorisant. X-cerberus est un autre inducteur de tête, décrit chez le xénope par le groupe de De Robertis (Los Angeles, CA, USA) ; l'injection de son ARN messager dans les embryons de xénope induit la formation de têtes ectopiques [5]. Il agirait lui aussi en inhibant la voie de transmission du signal Wnt [6]. Pour former la tête, il faudrait donc réprimer les voies de signalisation de Wnt et BMP qui

\footnotetext{
* Ils n'induisent pas siamois, cible précoce de la signalisation par la voie Wnt. Le gène dkk-1 agit donc en aval de siamois pour induire directement la formation de la tête, de pair avec l'expression de marqueurs neuraux, et en l'absence de formation de mésoderme.
}

coopèrent dans la régionalisation dorso-ventrale de l'axe de formation du corps: la mise en place de la tête a lieu «par défaut» de l'activation du signal Wnt.

\section{... et chez la souris - $m d k k-1$, et les autres}

L'homologue murin de $d k k-1, m d k k-1$, est exprimé chez la souris dès la gastrulation puis dans le mésoderme antérieur au stade du pli neural; il présente les mêmes propriétés d'induction que $d k k-1 \mathrm{chez}$ le xénope [2], c'est-à-dire la formation d'un axe secondaire antérieur. Tous deux agissent en inhibant la voie de transmission du signal BMP. Le groupe de De Robertis a rapporté récemment [3] le clonage et la caractérisation d'un homologue murin de Cerberus, qu'il avait initialement isolé chez le xénope [5]. L'ARNm mCer-like injecté dans des pôles animaux d'embryon de xénope est capable d'induire la formation d'un axe secondaire comportant des structures très antérieures, dont les glandes adhésives, révélatrices d'une activité anti-BMP. Enfin, C. Biben et al. (Victoria, Australie) ont très récemment décrit un autre membre de la famille Cerberus (mCerberus ou mCer-1) qui a la particularité d'être exprimé dans l'endoderme viscéral antérieur de l'embryon de souris [7], puis dans le mésendoderme, issu de la plaque nodale. Le facteur mCer-1 [7] comme mCer-like [3] induit la formation de structures antérieures. Ces facteurs agiraient, chez l'embryon de souris aussi, en inhibant la voie de transmission du signal Wnt [6]. $\mathrm{Au}$ vu du mode d'action de mdkk-1 et de mCerberus, apparaît à nouveau l'idée que pour antérioriser et établir un axe antéro-postérieur, il faut dégager une zone neutre dans les 
interactions des voies Wnt/BMP impliquées dans la régionalisation dorso-ventrale.

\section{Intervention \\ de l'endoderme viscéral antérieur}

Quel tissu, quels mécanismes sont capables d'établir les structures antérieures chez la souris? On sait déjà que la plaque nodale chez la Souris se comporte comme le nœud organisateur de Spemann chez le xénope. Elle est capable d'induire la formation d'axes secondaires, mais dépourvus de structures antérieures [8]. La souris présente donc aussi cette dichotomie dans l'organisation de l'axe antéro-postérieur.

L'endoderme viscéral est le feuillet primitif, à destin extra-embryonnaire, qui entoure l'œuf cylindre (figure 1). L'endoderme viscéral antérieur couvre l'épiblaste dans la région opposée au futur sillon primitif, avant la gastrulation, c'est-à-dire avant l'induction du mésoderme et la manifestation morphologique du pôle postérieur. Son intégrité est nécessaire au développement de la gastrulation. L'analyse de l'effet de mutations nulles de plusieurs gènes exprimés dans l'endoderme viscéral antérieur a montré leur implication dans la formation de la tête chez la souris (Otx2, HNF3 $\beta$, nodal). La combinaison d'endoderme viscéral «sauvage» et d'embryons mutants dans des embryons chimères permet de pallier l'effet de la mutation et à l'embryon de s'antérioriser [9]. Rosa Beddington a montré que l'expression du gène Hesx1 dans l'endoderme viscéral antérieur est indispensable pour activer ce même gène dans le neuroectoderme sous-jacent [10]. Le facteur mCerberus, dont les propriétés antériorisantes viennent d'être établies, est également exprimé dans l'endoderme viscéral antérieur suggérant que ce tissu extra-embryonnaire joue un rôle fondamental dans la spécification et l'organisation de la tête (figure 1). L'endoderme viscéral antérieur serait donc une source de signaux antériorisants indispensables à la poursuite normale du développement, tout en n'y participant pas per se; il joue un rôle déterminant dans la spécifica-

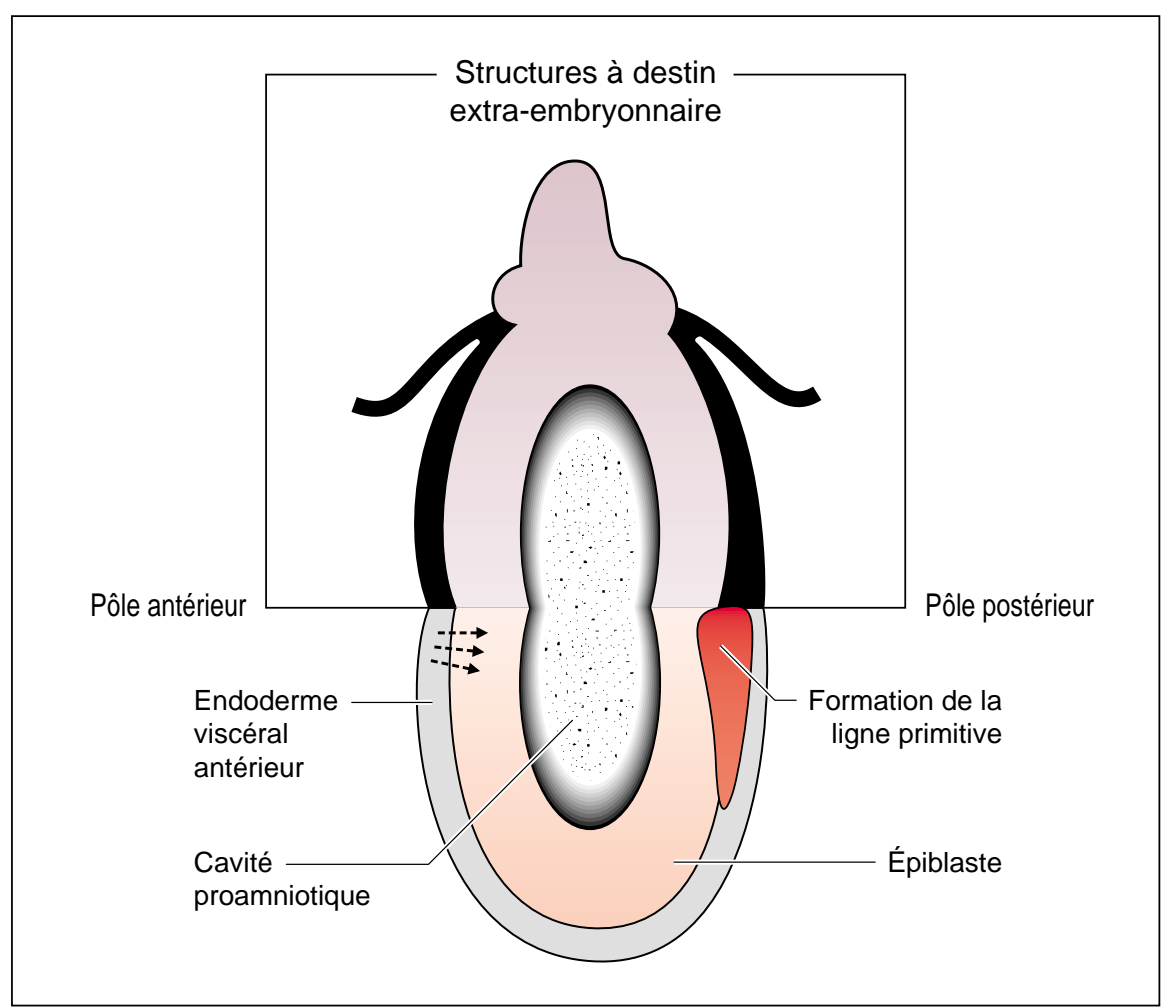

Figure 1. Embryon de souris au jour embryonnaire 6,5. L'œuf cyclindre est montré après ablation du sac pariétal. L'endoderme viscéral embryonnaire recouvre l'épiblaste (en rose) qui donnera tous les tissus embryonnaires. L'endoderme viscéral antérieur préexiste à la formation de la ligne primitive. Il lui est diamétralement opposé ; la ligne primitive se forme au jour 6,5 avec la délamination du mésoderme (en rouge) à la jonction entre tissus à destins embryonnaire et extra-embryonnaire. Son site définit le pôle postérieur de l'embryon. On peut remarquer que l'information initiale, spécifiant les premiers événements de régionalisation antérieure dans l'embryon et l'induction de la formation de la tête (indiquée par des flèches pointillées) provient d'un tissu extra-embryonnaire.

tion de l'axe antéro-postérieur de l'embryon de souris, bien avant la gastrulation et l'induction du mésoderme. L'induction de la tête, dans la spécification de l'axe antéro-postérieur, doit donc se concevoir dans le contexte de la régionalisation dorsoventrale et impliquerait, chez la souris, l'intervention d'un tissu extraembryonnaire.

M.O.O.

1. Lemaire $\mathrm{P}$, Kodjabachian $\mathrm{L}$. The vertebrate organizer: structure and molecules. Trends Genet $1996 ; 12: 525-31$.

2. Glinka A, Wu W, Delius H, Monaghan AP, Blumenstock C, Niehrs C. Dickkopf-1 is a member of a new family of secreted proteins and functions in head induction. Nature 1998; 391 : 357-62.

3. Belo JA, Bouwmeester T, Leyns L, Kertesz N, Gallo M, Follettie M, De Robertis EM. Cerberuslike is a secreted factor with neutralizing activity expressed in the anterior primitive endoderm of the mouse gastrula. Mech Dev 1997; 68: 45-57. 4. Honoré E, Hemmati-Brivanlou A. L'induction neurale chez les vertébrés: le cerveau par défaut. Med Sci 1997; 13: 192-200.

5. Bouwmeester T, Kim S, Sasai Y, Lu B, De Robertis EM. Cerberus is a head-inducing secreted factor expressed in the anterior endoderm of Spemann's organizer. Nature 1996; 382: 595-601. 6. Glinka A, Wu W, Onichtchouk D, Blumenstock C, Niehrs C. Head induction by simultaneous repression of Bmp and Wnt signalling in Xenopus. Nature 1997; 389: 517-9.

7. Biben C, Stanley E, Fabri L, Kotecha S, Rhinn $\mathrm{M}$, et al. Murine Cerberus homolog mCer-1: a candidate anterior patterning molecule. Dev Biol 1998; 194: 135-51.

8. Beddington RS. Induction of a second neural axis by the mouse node. Development 1994; 120 : 613-20.

9. Beddington RS, Robertson EJ. Anterior patterning in mouse. Trends Genet 1998; 14: 277-84.

10. Thomas PQ, Beddington RS. Anterior primitive endoderm may be responsible for patterning the anterior neural plate in mouse embryo. Curr Biol 1996; 6: 1487-96. (1)

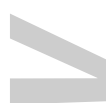

